\title{
Body composition at birth and height at 2 years: a prospective cohort study among children in Jimma, Ethiopia
}

\author{
Bitiya Admassu', Jonathan CK. Wells ${ }^{2}$, Tsinuel Girma ${ }^{3}$, Gregers S. Andersen ${ }^{4}$, Victor Owino ${ }^{5}$, Tefera Belachew ${ }^{1}$,
} Kim F. Michaelsen ${ }^{6}$, Mubarek Abera ${ }^{7}$, Rasmus Wibaek $^{4}$, Henrik Friis ${ }^{6}$ and Pernille Kæstel ${ }^{6}$

BACKGROUND: Low birth weight is associated with childhood stunting, but equivalent associations for birth body composition (BC) remain unknown. The aim of this study was to assess associations between birth $B C$ and height-for-age z-score (HAZ) at 2 years of age.

METHODS: In a prospective cohort study, fat mass (FM) and fat-free mass (FFM) were measured using air-displacement plethysmography within $48 \mathrm{~h}$ of birth. Linear regression models were applied to study the relationship between $B C$ at birth and HAZ at $24 \pm 3$ months.

RESULTS: A total of 268 children with height assessment at 2 years were included. Mean \pm SD HAZ at 2 years of age was $-1.2 \pm 1.2$, with $25.8 \%$ classified as stunted (HAZ $<-2 \mathrm{SD}$ ). FFM at birth was positively associated with HAZ at 2 years, independent of length at birth. When adjusted for potential confounders, HAZ at 2 years was 0.73 higher for each additional $\mathrm{kg}$ FFM at birth ( $\beta=0.73,95 \% \mathrm{Cl}$ : 0.08, 1.38). FM was not associated with $\mathrm{HAZ}$ at 2 years in any model.

CONCLUSION: The FFM component of birth weight, independent of length at birth, explained variability in HAZ at 2 years. Further studies are required to explore how changes in early infant BC are associated with linear growth.

D espite significant advances in child nutrition and health, under-nutrition remains one of the main public health challenges in low- and middle-income countries (1). Globally, around 159 million children $<5$ years are diagnosed with stunting (height-for-age $z$-score (HAZ) $<-2)(2)$. In Ethiopia, stunting affected 24 and $42 \%$ of children $<5$ years of age in urban and rural areas, respectively (3).

Stunting is a well-known risk factor for childhood mortality and impaired development $(4,5)$. It results in poor learning capacity and school performance during childhood, and reduced earning and work capacity during adulthood (4-6). This chronic nutritional problem occurs during the crucial period of pregnancy and in the first 2 years of life, the 'first 1,000 days', during which growth and nutrition have lifelong health implications $(4,7)$.

Stunting in childhood is the result of numerous circumstances and determinants, including antenatal and postnatal malnutrition (8), and is not uniformly understood $(4,8)$. Small size at birth, low maternal height, inappropriate feeding, and a high number of infections, especially diarrhea, have been associated with stunting $(9,10)$. Although numerous authors identified low birth weight as one of the risk factors for childhood stunting, body composition has received less attention. BC in children is now understood to be an indicator of current health status (11) and is associated with disease in later life $(12,13)$. In this regard, studies that investigate $\mathrm{BC}$ during childhood are important for a better understanding of stunting in children.

Presently, however, only a few studies have investigated the relation between $\mathrm{BC}$ at birth and linear growth faltering $(10,14-16)$. In those studies, body composition was assessed using simple anthropometric indices such as body weight, body mass index, and skinfold measurements, which may not be accurate and precise measurements of $\mathrm{BC}$ in children and may not adequately reflect total body fatness during infancy (17). To the best of our knowledge, no previous study has investigated $\mathrm{BC}$ as a predictor of HAZ.

It was recently shown in the Ethiopian Infant Anthropometry and Body Composition cohort (ABC) that neonates with the same birth weight had a large variation in FM and FFM (18). In addition, the study demonstrated that groups with lower birth weight, such as girls and first-born children, had reduced FFM but not significantly less FM. This indicates that studies using crude measurement techniques like body weight and body mass index might miss the potential effects of variation in $\mathrm{BC}$ on health outcomes.

In this analysis, we present findings from the 2-year followup of the iABC study. The aim of the study was to investigate the association between birth body composition and HAZ at 2 years of age.

\footnotetext{
${ }^{1}$ Department of Population and Family Health, College of Public Health and Medical Sciences, Jimma University, Jimma, Ethiopia; ${ }^{2}$ Childhood Nutrition Research Centre, UCL Institute of Child Health, London, UK; ${ }^{3}$ Department of Pediatrics and Child Health, College of Public Health and Medical Sciences, Jimma University, Jimma, Ethiopia; ${ }^{4}$ Steno Diabetes Center Copenhagen, Gentofte, Denmark; ${ }^{5}$ International Atomic Energy Agency, Vienna, Austria; ${ }^{6}$ Department of Nutrition, Exercise and Sports, University of Copenhagen, Copenhagen, Denmark; ${ }^{7}$ Department of Psychiatry, College of Public Health and Medical Sciences, Jimma University, Jimma, Ethiopia. Correspondence: Bitiya Admassu (bitiyaa@yahoo.com)

Received 16 November 2016; accepted 26 February 2017; advance online publication 7 June 2017. doi:10.1038/pr.2017.59
} 


\section{METHODS}

\section{Study Design, Setting, and Participants}

This prospective cohort study was conducted at Jimma University Specialized Hospital (JUSH) in Jimma town, Ethiopia, from January 2009 to August 2014.

Data for the present study were collected as a part of the iABC cohort study, which has been described in detail elsewhere $(18,19)$. Briefly, eligible mother-infant pairs were recruited among women who gave birth at the maternity ward at JUSH. Women and their newborns were eligible if they were living in Jimma town and the child was born at term (gestational age of $\geqslant 37$ weeks, using Ballard score assessment) with a birth weight $\geqslant 1,500 \mathrm{~g}$ and without congenital malformation. Women and newborns were examined within 48-h after birth. Newborns with serious medical conditions observed during baseline examination were not included. A total of 617 infants were recruited at birth. Of them, 268 had height measurements at 2 years and were analyzed in this study.

\section{Data Collection}

Anthropometric measurements. Length was measured in the supine position for children below 2 years, whereas height was measured in standing position for those aged 2 years and above according to WHO guidelines. Anthropometric measurements were taken at 2 years \pm 3 months. Length/height was measured in duplicate to the nearest $0.1 \mathrm{~cm}$ using a SECA 416 length board (SECA, Hamburg, Germany) and a SECA 213 portable stadiometer according to WHO recommendations (20), and the average was used for length/height analysis. For this paper, height was operationalized as HAZ based on the WHO growth standards (21), and stunting was defined as HAZ below minus two standard deviations $(-2 \mathrm{SD})$ from the median length/height for age of the reference population.

Birth weight, FM, and FFM were measured within $48 \mathrm{~h}$ after birth to the nearest $1 \mathrm{~g}$ using a Peapod ${ }^{\circledR}$, an infant air-displacement plethysmograph (ADP) specifically developed for measurement of infant BC (COSMED, Rome, Italy) (22). ADP is considered a fast, safe, precise, and accurate densitometry technique to assess $\mathrm{BC}$ in neonates and infants (23). The theory and standard operating procedures used in the present study have been described elsewhere (19). ADP is a two-component model that divides body weight into FFM and FM. FM consists of only fat and is free from water, whereas FFM includes protein, water, and mineral (osseous and non-osseous).

Questionnaire data at birth. Data were collected by trained research nurses using a structured and pre-tested questionnaire.

Information on maternal age and parental education were obtained. Data on socioeconomic status such as water quality, consumer durables (electricity, telephone, mobile, television, radio, refrigerator, stove, car, motor cycle, and electric injera mitad), and toilet facility were collected at the time of delivery.

Questionnaire data at 2 years of age. Hospitalization was assessed using the question "Was the child ever admitted to any health facilities for treatment since birth", and the response was recorded as yes/no.

Mothers were asked about the milk intake of their child within 1 week before the interview at 2 years of age. Milk intake of the child was described by total frequency of milk intake other than breast milk during the past 1 week. The response was categorized as not consumed, regularly consumed (1-6 days per week), or consumed every day (7 days).

\section{Data Handling and Statistical Analysis}

Data were double-entered using EpiData (version 3) (Odense, Denmark, 2003-2008). STATA/IC 12.1 (StataCorp, College Station, TX) was used for data analysis. The Zscore06 Stata command was used for the calculation of anthropometric $z$-scores using the 2006 WHO child growth standards (24).

Mean \pm SD was calculated for symmetric continuous variables. A wealth index was constructed from three overall socioeconomic categories: drinking water quality (four items, response 0 or 1), consumer durables (ten items, response 0 or 1 ), and toilet facility (five items, response 0 or 1 ). The wealth index score was generated from the data using principal components analysis (PCA); the first PCA factor that explained most of the variation was used to group study households into wealth quintiles (25). $\chi^{2}$ tests and $t$-tests were used to examine the differences between those included and those lost to follow-up. Simple and multiple linear regression analyses were performed. Linear regression analysis was applied to examine the association between body composition (FM or FFM) at birth as the main exposure variables and HAZ at 2 years of age as the outcome. The contribution of other covariates to HAZ at 2 years of age were evaluated by adding those variables to the linear regression models.

Separate multiple linear regression models for FFM and FM were developed (adjusting for early infant characteristics, cow milk intake, morbidity, and parental factors). Model 1: FFM or FM was included in the model as a continuous variable and birth length, sex, and current age were adjusted for. Model 2: same as model 1 but included cow milk intake, breastfeeding, and hospitalization. Model 3: same as model 2 but included maternal age, maternal education, and paternal education. Model 4 (fully adjusted model): same as model 3 but included parental wealth index.

Variables were considered for the multiple regression models if they were main exposure variables; potential confounding factors were identified a priori from the literature or affected the estimate of main exposure (birth FM and FFM). A $P$-value $<0.05$ was considered significant.

Scatter plots of the residuals vs. the independent variables and the predicted values were examined to determine whether there were patterns diverging from a normality distribution. Variance inflation factor (VIF) and tolerance test were used to test for the presence of multi-collinearity among the independent variables. VIF $<10$ was accepted. The results were presented using $\beta$-coefficients and $95 \%$ confidence intervals. Also, presence of interaction was checked between sex, wealth index, FM, and FFM, and a $P$-value $<0.05$ considered significant.

\section{Ethical Considerations}

Ethical approval was obtained from Jimma University, College of Public Health and Medical Science's Institutional Review Board (reference $\mathrm{RPO} / 56 / 2001)$. Written informed consent was obtained from each mother or guardian before commencing data collection. Participants who were unable to sign gave their thumb print. To maintain confidentiality, any identifiers were removed from the data before analysis. There were no risks associated with any of the examinations. Children found to be ill were referred for diagnosis and treatment at Jimma University Specialized Hospital.

\section{RESULTS}

Of 617 children recruited at birth, 271 (44\%) were followed up at 2 years. Of those, 268 (98.9\%) had a valid height measurement and were included in the study. A flow chart of the follow-up of study participants is presented in Figure 1.

The mean \pm SD gestational age was $38.9 \pm 1$ weeks. The mean \pm SD FM at birth was $0.2 \pm 0.2 \mathrm{~kg}$, whereas mean $\pm \mathrm{SD}$ FFM was $2.8 \pm 0.3 \mathrm{~kg}$ (Table 1 ). At follow-up, the mean $\pm \mathrm{SD}$ age of children was $24.3 \pm 0.8$ months. The mean \pm SD HAZ at 2 years of age was $-1.2 \pm 1.2$, and $25.8 \%(n=65)$ were stunted.

Nearly half of the mothers were between 20 and 24 years of age $(n=115,43.4 \%)$. Nearly two-thirds of mothers had completion of primary school as their highest educational level ( $n=167,62.3 \%$ ), and more than half of the fathers had primary school level as the highest $(n=153,59.1 \%)$ (Table 1). 


\section{Body composition at birth and height Articles}

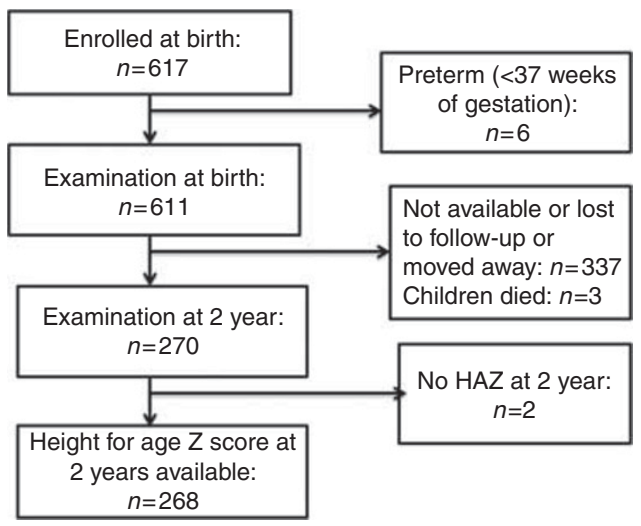

Figure 1. Flow chart of the study participants.

Table 1. Child and parental characterstics of 268 Ethiopian study participants at birth

\begin{tabular}{|c|c|}
\hline Variable & Mean \pm SD or $n(\%)$ \\
\hline \multicolumn{2}{|l|}{ Child characteristics at birth } \\
\hline Weight, kg & $3.1 \pm 0.4$ \\
\hline Low birth weight $(<2.5 \mathrm{~kg})$, (\%) & $20(7.5)$ \\
\hline Length, cm & $49.2 \pm 1.9$ \\
\hline Fat mass, kg & $0.2 \pm 0.2$ \\
\hline Fat-free mass, kg & $2.8 \pm 0.3$ \\
\hline Female sex, (\%) & $135(50.4)$ \\
\hline \multicolumn{2}{|l|}{ Parental characteristics at birth } \\
\hline Maternal age at delivery, year & $23.9 \pm 4.5$ \\
\hline \multicolumn{2}{|l|}{ Maternal education, (\%) } \\
\hline No school & $22(8.2)$ \\
\hline Primary & $167(62.3)$ \\
\hline$\geqslant$ Secondary & $79(29.5)$ \\
\hline \multicolumn{2}{|l|}{ Paternal education, (\%) } \\
\hline No school & $8(3.2)$ \\
\hline Primary & $153(59.1)$ \\
\hline$\geqslant$ Secondary & $98(37.8)$ \\
\hline
\end{tabular}

Concerning household possessions, the majority of parents had electricity $(n=258,96.3 \%)$, radio ( $n=243,90.7 \%)$, mobile $(n=196,71.1 \%)$, and television ( $n=182,67.9 \%)$. Only 3 (1\%) parents who were ranked at the 5 th level (richest) on the wealth index did not own a television, compared with $51(82.3 \%)$ parents who were ranked at level one (poorest).

\section{Predictors of Height-for-Age z-score at 2 years}

Body composition. Separate multiple linear regression models are presented for FFM and FM. FFM at birth was associated with HAZ at 2 years in both simple (Table 2) and multiple linear regression analysis (Table 3 ). When adjusted for neonatal factors (model 1), FFM was associated with HAZ at 2 years $(\beta=0.68,95 \% \mathrm{CI}: 0.06,1.31)$. After adjusting for current child milk intake, hospitalization, and breastfeeding at 2 years, the estimate of FFM was stronger (model 2). HAZ at 2 years was 0.77 higher for each additional $\mathrm{kg}$ FFM at birth $(\beta=0.77,95 \%$ CI: $0.13,1.41)$. Adjusting for maternal age, parental education further increased the estimate of FFM (model 3) ( $\beta=0.85,95 \%$ CI: 0.20, 1.42). Further adjustment for parental wealth index in model 4 reduced the estimate of FFM, although the associations with birth body composition were still significant $(\beta=0.73,95 \% \mathrm{CI}: 0.08,1.38)$. FM was not associated with HAZ at 2 years in any of the four models, and the direction of the non-significant relation was negative with HAZ. The association between FFM and HAZ was not modified by sex or wealth index ( $P$ for interaction $>0.05$ ).

The association between FFM at birth and HAZ at 2 years of age was also examined by excluding length at birth from the model. Unadjusted for length, the association between FFM at birth and HAZ at 2 years was stronger and highly significant (data not shown).

Furthermore, in the absence of FFM and FM, association between birth weight and HAZ at 2 years was examined. However, birth weight was significant only in simple linear regression, whereas it was not significantly associated with HAZ when adjusted for potential confounders in subsequent models (data not shown).

In addition, we have analyzed the association between BMI-for-age $z$-score (BMIZ) with HAZ at 2 years as a proxy for body composition. BMIZ was positively associated with HAZ at 2 years in simple linear regression and lost significance when adjusted for potential confounders in subsequent models (data not shown).

In addition to FFM, in the fully adjusted model, being female was associated with 0.61 higher HAZ at 2 years $(\beta=0.61,95 \% \mathrm{CI}: 0.33,0.89)$. Each $\mathrm{cm}$ in length at birth was associated with $0.12(\beta=0.12,95 \% \mathrm{CI}: 0.01,0.22)$ larger HAZ at 2 years. A month increase in the child's age at 2 years was associated with a 0.24 lower $\operatorname{HAZ}(\beta=-0.24,95 \%$ CI: -0.41 , -0.07). These associations were maintained in all models (data not shown). HAZ was higher in children who received cow milk every day compared with those who did not during the previous 7 days $(\beta=0.42,95 \%$ CI: $0.11,0.73)$. This association was maintained in the succeeding model but not in the final model, where we adjusted for parental wealth index. Higher parental wealth index score was also positively associated with HAZ $(\beta=0.14,95 \% \mathrm{CI}: 0.03,0.26)$.

\section{DISCUSSION}

FFM but not FM was consistently positively associated with HAZ at 2 years. This association was independent of length at birth and parental wealth.

Although no previous studies have explored the relationship between birth body composition and HAZ, a number of earlier studies had identified low birth weight as a predictor of 


\section{Articles | Admassu et al.}

linear growth $(10,15)$. FFM rather than FM accretion during gestation might explain the association between birth weight and HAZ at 2 years of age. A higher FFM at birth might indicate healthy nutritional intake of the mother during

Table 2. Univariate linear regression analysis between birth body composition and other covariates, and child height-for-age $z$-scores at 2 years of age in 268 Ethiopian children

\begin{tabular}{lcc}
\hline & $\beta(95 \% \mathrm{Cl})$ & $P$-value \\
\hline Birth characteristics & & \\
Fat-free mass, kg & $1.16(0.72,1.61)$ & $<0.001^{*}$ \\
Fat mass, kg & $1.01(0.13,1.88)$ & $0.02^{\dagger}$ \\
Length, cm & $0.22(0.15,0.29)$ & $<0.001^{*}$ \\
Female sex $(n=135)$ & $0.35(0.07,0.63)$ & $0.01^{\dagger}$
\end{tabular}

Child characteristics

7-day cow milk intake

Not at all $(n=135)$

Ref

$1-6$ days $(n=31)$

$0.26(-0.19,0.71)$

0.25

Every day $(n=101)$

$0.61(0.31,0.91) \quad<0.001^{*}$

Hospitalization since birth,

$-0.38(-1.82,0.07)$

0.10

Yes $(n=30)$

Breastfeeding at 2 years, Yes $(n=169)$

$-0.12(-0.42,0.17) \quad 0.42$

\section{Parental factors}

Maternal age at delivery, year

$0.02(-0.01,0.05)$

0.29

Maternal education

No school $(n=22)$

Ref

Primary $(n=167)$

$\geqslant$ Secondary $(n=79)$

Paternal education

No school $(n=8)$

Primary $(n=153)$

$\geqslant$ Secondary $(n=98)$

Parental wealth index score

${ }^{*} P<0.01$, significant values

${ }^{+} P<0.05$, significant values

Data are given as regression coefficients ( $\beta$ ) and $95 \%$ confidence limits. pregnancy, which in turn may have positive effects on fetal growth and newborn health.

Barker et al. (26) have proposed that poor fetal nutrition could reduce the concentration of insulin-like growth factor type 1 (IGF-1) and thereby undermine the growth of muscle mass. Thus, a lower concentration of IGF-1 could be one explanation why we find in the present study that reduced birth weight is particularly related to reduced FFM. A few other studies have indicated that poor growth during fetal life and infancy could permanently constrain FFM, which could adversely affect later growth $(27,28)$. There are a few studies showing that low FFM in early life predicts insulin resistance $(29,30)$. Maternal dietary restriction may affect fetal growth directly via placental nutrient availability (31), and also indirectly via the fetal endocrine system and epigenetic modulation of gene expression $(32,33)$.

In our study, FM at birth was not associated with HAZ at 2 years in either the univariate or the multivariable analysis. However, the direction of the relationship with HAZ was negative. It is likely that fat stores at birth might represent a store of energy to fund infant growth, but we did not find any association. Although FM was not associated with HAZ in the current study, a study conducted by Carberry et al. (34) in Australia showed that body fat percentage was a better predictor of neonatal morbidity than birth weight percentiles and performed better in identifying undernourished neonates. However, using body fat percentage as an indication of fat mass properties is not advisable as the fat percentage includes both the properties of fat mass and total body mass in the same measurement (17).

FFM and FM have distinct growth patterns during gestation. FFM accretion occurs all the way through pregnancy, whereas more than $90 \%$ of the FM accretion occurs during late gestation and shows an accelerated growth (35). This difference in fetal tissue accretion might explain the association between FFM and HAZ as well as the lack of association between FM and HAZ.

Although reference values of FFM and FM could be useful in a clinical setting and field surveys, in recent year few studies have provided population-specific reference data. In fact, in 2013 reference data were published based on a subsample of the population included in the current

Table 3. Multiple linear regression analysis examining the independent associations between birth fat-free mass and fat mass on child heightfor-age $z$-score at 2 years of age

\begin{tabular}{|c|c|c|c|c|}
\hline & \multicolumn{4}{|c|}{$\beta(95 \% \mathrm{Cl})$} \\
\hline & Model-1 ${ }^{a}$ & Model- $2^{\mathrm{b}}$ & Model-3c & Model-4 ${ }^{d}$ \\
\hline Fat-free mass (kg) & $0.68(0.06,1.31)^{\dagger}$ & $0.77(0.13,1.41)^{\dagger}$ & $0.85(0.20,1.50)^{\dagger}$ & $0.73(0.08,1.38)^{\dagger}$ \\
\hline \multicolumn{5}{|c|}{$\begin{array}{l}{ }^{+} P<0.05 \text {, significant values. } \\
\text { a Newborn measurements (FFM or FM, length at birth, and sex of the child) and child age at } 2 \text { years. } \\
{ }^{\circ} \text { Model } 1+2 \text { years' measurements (cow milk intake at } 2 \text { years, breastfeeding at } 2 \text { years, hospitalization since birth). } \\
c^{c} \text { Model } 2+\text { parental characteristics (baseline maternal age, maternal education and paternal education). } \\
\text { dModel 3+parental wealth index. }\end{array}$} \\
\hline
\end{tabular}




\section{Body composition at birth and height Articles}

manuscript (19), and the progression in the development of widely applicable reference data was described (36). However, as opposed to weight and height, there is no globally applicable standard available from WHO for FM and FFM.

We have also examined the association between birth weight and HAZ at 2 years. However, birth weight was not significantly associated with HAZ when adjusted for potential confounders in subsequent models. Similarly, association between BMIZ at birth and HAZ at 2 years was examined. However, BMIZ at birth lost significance when adjusted for potential confounders in the subsequent models. These findings show the importance of using FFM and FM measurements rather than a simpler anthropometric measure like birth weight and BMIZ. A study conducted by Andersen et al. (18), in the same cohort, showed a marked variation in FM and FFM for children with the same birth weight. This finding indicates that studies using crude measurement techniques like body weight might miss the potential effects of variation in body composition on health outcomes.

Most of the previously identified correlates also showed a statistically significant association with HAZ in this study. Female sex, birth length, and parental wealth had a positive association with HAZ, whereas child age had a negative association. Females had higher HAZ at 2 years, which is corroborated by previous studies conducted in Indonesia, Bangladesh, Bhutan, and India $(37,38)$ and by a meta-analysis of studies from sub-Saharan Africa (39).

In our study, length at birth was positively associated with $\mathrm{HAZ}$ at 2 years. This observation is consistent with a previous study conducted in Amazon, Brazil (16), and a review from five cohort studies in low- and middle-income countries (40). The finding implies that length at birth is an important predictor of linear growth, revealing its importance for interventions to address intrauterine growth failure. Nevertheless, FFM at birth predicted HAZ at 2 years independent of length at birth.

Furthermore, HAZ at 2 years was significantly higher in children with a higher parental wealth index score. This finding is consistent with previous studies conducted in Bhutan, Eastern Himalayas and India $(37,38)$.

Our study has limitations. Maternal pre-pregnancy and pregnancy-related factors were not assessed. Potential confounding by complementary feeding was also not addressed. The sample size was relatively small and might result in low statistical power. Information related to hospital admission could have been subject to recall bias. Further, a crude measure of morbidity was used and further adjustment for less serious morbidities could not be made, although they contribute a lot to growth faltering. Considerable loss to follow-up is also a limitation. However, children included in the analyses were not statistically different from those who were not included with respect to sex, age, birth order, length, FFM and FM at birth, parental education, and parental wealth index (Supplementary Table S1 online). Functional anomalies were not assessed, which could potentially confound the association between $\mathrm{BC}$ and height. Among the main strengths of the study is its prospective longitudinal design, as well as the use of an air-displacement plethysmograph to assess body composition at birth, which is a precise and accurate method for assessing fat and fat-free mass in infants (23).

In conclusion, our study suggests that FFM at birth was associated with $\mathrm{HAZ}$ at 2 years. In contrast, birth FM was not a correlate of HAZ at 2 years of age. This finding is relevant for future research studies that are using birth weight as the outcome. It suggests that future health interventions may have greatest benefit for child growth if they improve FFM rather than FM during fetal life.

We recommend studies to better understand which part of the FFM mediates this association. Studies looking at body composition with height by including maternal pre-pregnancy and pregnancy-related factors in detail might be needed. Follow-up studies might be required to explore how early infant body composition growth pattern is associated with linear growth. Furthermore, additional studies are required to explore how early life body composition is related to later under-nutrition, childhood obesity, and later susceptibility to non-communicable diseases.

\section{SUPPLEMENTARY MATERIAL}

Supplementary material is linked to the online version of the paper at http://www.nature.com/pr

\section{ACKNOWLEDGMENTS}

We sincerely thank the supervisors, research nurses and respondents, and Jimma University Specialized Hospital. We would like to thank Jimma University for giving us permission to conduct the study and publish the result.

\section{AUTHOR CONTRIBUTIONS}

The authors' responsibilities were as follows-BA, HF, JCKW, TG, GSA and PK designed the study; BA, GSA, PK, MA and RW supervised the data collection; BA. analyzed the data and drafted the manuscript, and had primary responsibility for the final content of the manuscript. All authors contributed to the manuscript revisions and read the final manuscript and approved for submission.

\section{STATEMENT OF FINANCIAL SUPPORT}

Danish Council for Strategic Research-Programme Commission on Food and Health; Danida through the Consultative Research Committee for Development Research (104.Dan.8-1207).

Disclosure: The authors declare no conflict of interest.

\section{REFERENCES}

1. Black RE, Allen LH, Bhutta ZA, et al. Maternal and child undernutrition: global and regional exposures and health consequences. Lancet 2008;371: 243-60.

2. Research Institute (IFPRI) IFP Global Nutrition Report 2016 From Promise to Impact Ending Malnutrition by 2030. Washington, DC: International Food Policy Research Institute, 2016. Available at http://ebrary.ifpri.org/ cdm/ref/collection/p15738coll2/id/130354. Accessed 1 September 2016.

3. Central Statistical Agency [Ethiopia]. Ethiopia Mini Demographic and Health Survey 2014. Addis Ababa, Ethiopia, 2014. Available at http://www.measuredhs.com/pubs.pdf. Accessed 19 August 2015.

4. Black RE, Victora CG, Walker SP, et al. Maternal and child undernutrition and overweight in low-income and middle-income countries. The Lancet 2013;382:427-51. 


\section{Articles 1 Admassu et al.}

5. Dewey KG, Begum K. Long-term consequences of stunting in early life. Matern Child Nutr 2011;7:5-18.

6. Allen L, Gillespie S. What works? A Review of the Efficacy and Effectiveness of Nutrition Interventions. Geneva: United Nations, Administrative Committee on Coordination, Sub-Committee on Nutrition, 2001.

7. Bhutta ZA, Salam RA. Global nutrition epidemiology and trends. Ann Nutr Metab 2012;61:19-27.

8. Prendergast AJ, Humphrey JH. The stunting syndrome in developing countries. Paediatr Int Child Health 2014;34:250-65.

9. Iannotti LL, Zavaleta N, León Z, Caulfield LE. Growth and body composition of Peruvian infants in a periurban setting. Food Nutr Bull 2009;30:245-53.

10. Padonou G, Le Port A, Cottrell G, et al. Factors associated with growth patterns from birth to 18 months in a Beninese cohort of children. Acta Trop 2014;135:1-9.

11. Wells JCK. Measuring body composition. Arch Dis Child 2005;91: 612-7.

12. Dencker M, Wollmer P, Karlsson MK, Lindén C, Andersen LB, Thorsson O. Body fat, abdominal fat and body fat distribution related to cardiovascular risk factors in prepubertal children. Acta Paediatr 2012;101:852-7.

13. Gishti O, Gaillard R, Durmus B, et al. Body mass index, total and abdominal fat distribution and cardiovascular risk factors in school-age children. Pediatr Res 2015;77:710-8.

14. Hanieh S, Ha TT, De Livera AM, et al. Antenatal and early infant predictors of postnatal growth in rural Vietnam: a prospective cohort study. Arch Dis Child 2015;100:165-73.

15. Medhin G, Hanlon C, Dewey M, et al. Prevalence and predictors of undernutrition among infants aged six and twelve months in Butajira, Ethiopia: the P-MaMiE Birth Cohort, BMC Public Health 2010;10:27.

16. Lourenço BH, Villamor E, Augusto RA, Cardoso MA. Determinants of linear growth from infancy to school-aged years: a population-based follow-up study in urban Amazonian children. BMC Public Health 2012;12:265.

17. Wells JC. A critique of the expression of paediatric body composition data. Arch Dis Child 2001;85:67-72.

18. Andersen GS, Girma T, Wells JCK, Kæstel P, Michaelsen KF, Friis H. Fat and fat-free mass at birth: air displacement plethysmography measurements on 350 Ethiopian newborns. Pediatr Res 2011;70:501-6.

19. Andersen GS, Girma T, Wells JC, et al. Body composition from birth to 6 mo of age in Ethiopian infants: reference data obtained by airdisplacement plethysmography. Am J Clin Nutr 2013;98:885-94.

20. World Health Organization Training Course on Child Growth Assessment. Geneva: WHO, 2008.

21. WHO Multicentre Growth Reference Study Group WHO Child Growth Standards: Length/ Height-for-Age, Weight-for-Age, Weight-for-Length, Weight-for-Height and Body Mass Index-for-Age: Methods and Development. Geneva: World Health Organization, 2006.

22. Urlando A, Dempster P, Aitkens S. A new air displacement plethysmograph for the measurement of body composition in infants. Pediatr Res 2003;53:486-92.

23. Ellis KJ, Yao M, Shypailo RJ, Urlando A, Wong WW, Heird WC. Bodycomposition assessment in infancy: air-displacement plethysmography compared with a reference 4-compartment model. Am J Clin Nutr 2007;85:90-5.

24. Lorey J zscore06: Stata Command for the Calculation of Anthropometric z-Scores Using the 2006 WHO Child Growth Standards, 2006. Available at http://www.stata.com/statalist/archive/2011-04/msg01386.html. Accessed 31 August 312015.

25. Arimond M, T. Ruel M. International Food Policy Research Institute Report: Progress in Developing an Infant and Child Feeding Index: an Example Using The Ethiopia Demographic and Health Survey 2000, 2002. http://www.ifpri.org/publication/progress-developing-infant-andchild-feeding-index-0. Accessed 19 March 2016.

26. Barker DJ, Bull AR, Osmond C, Simmonds SJ. Fetal and placental size and risk of hypertension in adult life. BMJ 1990;301:259-62.

27. Wells JCK. The programming effects of early growth. Early Hum Dev 2007;83:743-8.

28. Longo S, Bollani L, Decembrino L, Di Comite A, Angelini M, Stronati M. Short-term and long-term sequelae in intrauterine growth retardation (IUGR). J Matern Fetal Neonatal Med 2013;26:222-5.

29. Joglekar CV, Fall CHD, Deshpande VU, et al. Newborn size, infant and childhood growth, and body composition and cardiovascular disease risk factors at the age of 6 years: the Pune Maternal Nutrition Study. Int J Obes 2007;31:1534-44.

30. Burrows R, Correa-Burrows P, Reyes M, Blanco E, Albala C, Gahagan S. Healthy Chilean adolescents with HOMA-IR $\geq 2.6$ have increased cardiometabolic risk: Association with Genetic, Biological, and Environmental Factors. J Diabetes Res 2015;2015:1-8.

31. Thornburg K, O'Tierney $\mathrm{P}$, Louey $\mathrm{S}$. The placenta is a programming agent for cardiovascular disease. Placenta 2010;31:S54-9.

32. Jansson T, Powell TL. Role of the placenta in fetal programming: underlying mechanisms and potential interventional approaches. Clin Sci 2007;113:1-13.

33. Gabory A, Attig L, Junien C. Developmental programming and epigenetics. Am J Clin Nutr 2011;94:1943S-52S.

34. Carberry AE, Raynes-Greenow CH, Turner RM, Askie LM, Jeffery HE. Is body fat percentage a better measure of undernutrition in newborns than birth weight percentiles? Pediatr Res 2013;74:730-6.

35. Haggarty P. Placental regulation of fatty acid delivery and its effect on fetal growth-a review. Placenta 2002;23:S28-38.

36. Wells JCK. Toward body composition reference data for infants, children, and adolescents. Adv Nutr Int Rev J 2014;5:320S-9S.

37. Aguayo VM, Badgaiyan N, Paintal K. Determinants of child stunting in the Royal Kingdom of Bhutan: an in-depth analysis of nationally representative data: Determinants of child stunting in Bhutan. Matern Child Nutr 2015;11:333-45.

38. Aguayo VM, Nair R, Badgaiyan N, Krishna V. Determinants of stunting and poor linear growth in children under 2 years of age in India: an indepth analysis of Maharashtra's comprehensive nutrition survey: child stunting in Maharashtra, India. Matern Child Nutr 2016;12:121-40.

39. Wamani H, Åstrøm AN, Peterson S, Tumwine JK, Tylleskär T. Boys are more stunted than girls in sub-Saharan Africa: a meta-analysis of 16 demographic and health surveys. BMC Pediatr 2007;7:17.

40. Stein AD, Wang M, Martorell R, et al. Growth patterns in early childhood and final attained stature: data from five birth cohorts from low- and middle-income countries. Am J Hum Biol 2010;22:353-9. 